\title{
Introduction: Attrition and second language acquisition
}

Monika S. Schmid ${ }^{1}$ and Barbara Köpke ${ }^{2}$

${ }^{1}$ University of Groningen, ${ }^{2}$ Université de Toulouse - Le Mirail

Traditionally, the study of language attrition — roughly defined as language 'loss' in bilingual individuals ${ }^{1}$ - - has often been regarded as a subfield of language contact, language change and language death rather than as a matter of language acquisition and bilingualism. This categorization is reflected in the fact that early collections on the topic of language attrition, such as Weltens, de Bot and van Els (1986) or Seliger and Vago (1991) contained studies of both attrition settings and intergenerational change in (endangered) minority languages, such as e.g. Pennsylvania German (Huffines 1991), Breton (Dressler 1991) or in language contact situations in general (Boyd 1986). 'Change' or ‘deterioration' in an existing, full-fledged linguistic system probably seemed too unlike its acquisition from scratch to warrant the localization of attrition studies within the bilingualism/acquisitional paradigm.

Grosjean's influential manifesto (Grosjean 1989), declaring that the 'bilingual is not two monolinguals in one person' went some way towards changing this state of affairs. He argues strongly in favour of a wholistic view on bilingualism, where linguistics will "no longer examine one of the bilingual's languages without examining the other; rather we will study how the bilingual structures and uses the two languages, separately or together, to meet his or her everyday communicative needs” (Grosjean 1989: 13). This change in paradigm and findings such as those from Flege's (1987) seminal study which presented evidence of a phonological divergence from the native norm in both languages of experienced bilinguals, began a process wherein language attrition came to be seen as an individual, psycholinguistic phenomenon that is the outcome of "the coexistence and constant interaction of the two languages” (Grosjean 1989: 6).

As the study of language attrition developed, it became evident that predictions made from the perspective of language change, such as a simplification in case systems or changes in underlying word order, were not borne out by the data (e.g. Schmitt 2004; Schmid 2002). Such reductions are confined to situations of acquisition where the input is restricted to certain contexts and/or does not conform entirely to the native standards, as is the case in long-term intergenerational change in contact settings.

Furthermore, cumulative evidence from a large range of investigations of language attrition (for an overview, see Köpke \& Schmid, this volume) has demonstrated that the differences between monolinguals and attriters, while interesting in nature, affect only a very small proportion of the total

\footnotetext{
${ }^{1}$ Where not explicitly stated otherwise, the present paper and all other contributions in the present collection will assume 'attrition' or 'attriter' to refer to late bilinguals, e.g. to individuals who grew up predominantly monolingually (with the possible exception of some instructed foreign language experience, as is the standard
} 
number of contexts. Schmid (forthcoming) proposes that for those phenomena where evidence for attrition has been found at all, it usually affects less than $5 \%$ of all occurrences. This suggests that attrition is the outcome of two systems of linguistic knowledge interacting, to some degree, in language processing during production and compréhension, and does not change the underlying knowledge system. As such, attrition is a psycholinguistic phenomenon that affects individual linguistic behaviour, and thus of an entirely different order than the slow processes of change or deterioration that occur on the societal level, mainly in the transmission from one generation to the next, which can affect overall grammatical systems and even lead to the disappearance of languages. It thus became generally accepted that a more relevant correlate for language attrition is second language acquisition, and that attrition studies can not only profit from the application of theories and principles of bilingual development but also serve to validate them to some extent (see Köpke \& Schmid, this volume). In particular, this contribution bears on the question of whether differences in bilingual and monolingual data (whether for production or for comprehension) can be located at the level of representation or at the level of access, a question that is closely linked to the competence/performance debate. The notions of underlying knowledge on the one hand and its application under the pressure of on-line speech production and comprehension on the other vary greatly across different linguistic frameworks. The question how ‘deep’ attrition can affect this knowledge therefore has different implications for different linguistic frameworks: for example, traditions which assume the presence of some kind of innate faculty for the acquisition of grammar will come to different predictions on language loss (assuming it to be constrained by underlying and universal principles) than researchers who see acquisition (and thus attrition) as usage-based. In the former approaches, it is now commonly accepted that late attrition - that is, among speakers for whom the onset of immersion in an L2 context was located after puberty - is confined to interface phenomena and does not affect narrow syntax (e.g. Sorace 2005). In more psycholinguistically oriented traditions, the questions focus less on the restructuring of underlying knowledge and more on the degree to which access to this knowledge becomes compromised. Relevant questions in this context are whether a speaker can still access a certain structure or lexical item for the purpose of production, and if this is no longer possible, is the structure/item still available for triggered recall in comprehension (e.g. Hansen 2011; Paradis 2007). In this context, issues such as different types of memory (procedural/declarative), distributional frequency and the amount of exposure a speaker retains from his/her L1 are relevant.

However, apart from these discipline-related distinctions in how the language faculty and its application are viewed, converging findings from attrition studies suggest that once the L1 system has been acquired and has stabilized - a process that is usually assumed to be completed around age 12

in most countries nowadays), and where the onset of bilingualism and attrition is located at the moment of migration, which takes place after puberty. 
(see Köpke \& Schmid, this volume) - this system, in itself, is not vulnerable to attrition any longer. This stability arguably allows attrition studies to provide valuable insights into the 'representational deficits’ debate in second language acquisition (e.g. Hawkins \& Hattori 2006; Snape, Leung \& Sharwood Smith 2009), as they can provide baseline data which are affected by crosslinguistic transfer, but not by representational deficits. It is therefore important that research on bilingualism fully recognizes that Grosjean's statement does not mean that a bilingual is a monolingual and a half, but that she or he is different from the monolingual in both language systems, and that it is the comparison of the system acquired from birth and the system acquired later in life that can provide valid answers to such questions.

The studies collected here address some of the issues that are briefly touched on above. The first paper, by Köpke and Schmid, provides an overview of the state of the art in attrition research, with special attention to the delimitation of the attrition field with respect to other research fields such as intergenerational language shift and language contact situations, incomplete acquisition and bilingualism effects. The paper then proposes a discussion of a variety of neuropsycholinguistic factors involved in attrition and describes some psycholinguistic manifestations of the attrition process. The point made here is that attrition of L1 in late bilinguals is a primarily psycholinguistic phenomenon, reflecting the competition between two languages with different activation states. Following this are two papers on the attrition of L1 Turkish and two on the attrition of L1 Spanish. Gürel \& Yllmaz investigate the binding properties of overt and null subject pronouns in L1 Turkish. The experiment reported here replicates Gürel's earlier (2002, 2004) investigation of this phenomenon in an L2 English setting in Canada. For the present study, both first and second generation Turks in the Netherlands are investigated. There are important differences between the populations examined in the precursor study and in the results presented here, as Turks in the Netherlands represent a much larger migrant group and tend to live in rather more close-knit communities. This implies that the transmission rates of the $\mathrm{L} 1$ to the subsequent generation(s) are higher than in the North American context. The findings from both populations in the Netherlands (attriters and heritage speakers) are very similar to the ones reported in Gürel (2002, 2004): all bilingual groups have some degree of restructuring with respect to the interpretation of overt and null subject pronouns in comparison to a monolingual control group. Of particular relevance is the finding that in those cases where no competing form exists in the L2 (the overt pronoun kendisi and the null pronoun, which for the controls are largely ambiguous between a bound and a disjoint reference), the bilinguals appear to have a tendency to revert to the canonical interpretation. This is interpreted as an emergent grammatical preference pattern among the bilinguals which is UG-compatible. These patterns are found to a similar degree in the responses from first and second generation speakers, as well as from speakers with English and Dutch as L2. Gürel and Yllmaz conclude that age at onset may not have played a predominant role in their sample, which may to some extent be the outcome of the high vitality that Turkish enjoys in the Netherlands. 
The paper by Yllmaz studies a similar population of attriters: late bilingual Turks in the Netherlands. She investigates to what degree syntactically complex sentence patterns, namely the various types of embedding used in Turkish, survive across long periods of residence in an L2 setting. Based on previous research she ranks the different structures used to form embeddings in the order of morphological complexity (that is, by the number of inflectional processes necessary to form each type). She finds that, compared to predominantly monolingual native speakers in Turkey, the attriters overuse only one of these types, but that this is the most morphologically complex one. Extralinguistic factors, such as length of residence, amount of L1 use, cultural affiliation and proficiency in L2 are shown not to have predictive power for individual variation; it is only the level of education that plays some role.

The paper by Montrul addresses one of the topics that has gained in importance in language attrition research since the seminal study by Pallier et al (2003), namely language loss and residual knowledge of the birth language in international adoptees (see also Schmid, forthcoming). She presents the case study of a woman who was adopted from Guatemala by US-American parents, who strongly discouraged any use of Spanish. In high school and later as a young adult, however, she attempted to re-learn her birth language and now makes frequent use of it. Investigating the behaviour of this bilingual on a variety of morphosyntactic variables, Montrul asks the intriguing question of whether her previous, interrupted experience with the language was able to give her a substantial advantage over other learners who have no such early exposure. She concludes that the interruption of input in the birth language which international adoptees experience does have drastic consequences for their potential to fully develop the birth language, but that age is a very important factor in this matter. Retention and facilitation in re-learning are further strongly affected by attitudes and motivation (evidently an extremely complex matter for adoptees in general and international adoptees in particular).

Lastly, Perpiñán addresses another question that has been controversially discussed in language attrition research, namely the issue of whether attrition is strictly confined to interface phenomena, or whether it can also affect core syntax. Based on a group of Spanish L1 residents in the US (who use English on a daily basis), she does find some evidence for emerging optionality in production in a type of subject-verb inversion construction which is pragmatically constrained, but not in contexts where it is purely syntactic. These findings provide support for Sorace's (2005) Optionality at the Interfaces Hypothesis. Receptive knowledge and response time, on the other hand, appear to be unaffected.

On the whole, these contributions demonstrate that research in attrition can contribute to shed light on some of the core questions in second language acquisition research such as the critical period debate, the representational deficit issue, the interface hypothesis, etc. Furthermore, several of the contributions show that the psycholinguistic dimension of the attrition phenomenon is not restricted to 
lexical retrieval difficulties, but can also affect for example the processing of syntax in specific domains. 


\section{Attrition et acquisition d'une langue seconde}

Monika S. Schmid ${ }^{1}$ et Barbara Köpke ${ }^{2}$

${ }^{1}$ University of Groningen, ${ }^{2}$ Octogone-Lordat (EA 4156), Université de Toulouse - Le Mirail

Dans le passé, l'étude de l'attrition d'une langue — définie grossièrement comme la « perte » d'une langue par un individu bilingue ${ }^{2}$ — a le plus souvent été associée aux recherches dans le domaine du contact des langues, du changement linguistique et de la mort des langues et non tellement à celle sur l'acquisition des langues et le bilinguisme. Cette association trouve son origine probablement dans le fait que les premières publications collectives sur ce sujet - p. ex. Weltens, de Bot et van Els (1986) ou Seliger et Vago (1991) - rassemblent des contributions tant sur l'attrition que sur des changements linguistiques intergénérationnels au niveau de langues minoritaires en danger, comme par exemple l'allemand de Pennsylvanie (Huffines 1991), le breton (Dressler 1991) ou dans des situations de contact en général (Boyd 1986). On estimait probablement que le « changement » ou la « détérioration » d’un véritable système linguistique déjà en place devait être trop différent de l'acquisition d'un tel système à partir de zéro pour inclure l'étude de l'attrition dans le paradigme bilinguisme/acquisition.

Un pas important vers une évolution de cette appréciation vient du célèbre manifeste de Grosjean (1989) déclarant que le bilingue n’est pas deux monolingues en une personne. Grosjean défend une vision holistique du bilinguisme où les chercheurs ne vont plus « examiner l'une des langues $d u$ bilingue sans tenir compte de l'autre mais plutôt étudier comment le bilingue structure et utilise ses langues, séparément ou ensemble, afin de répondre aux besoins communicatifs de la vie de tous les jours » (Grosjean 1989 : 13). Ce changement dans l'approche du bilinguisme ainsi que des résultats comme ceux de l'étude phare de Flege (1987) démontrant que les deux langues d'un bilingue expérimenté divergent des normes natives respectives des deux langues, ont déclenché un processus durant lequel on a commencé à considérer l'attrition comme un phénomène individuel, psycholinguistique qui est le résultat de « la coexistence et de l'interaction constante entre deux langues » (Grosjean 1989 : 6).

Lors du développement des études sur l’attrition il s’est révélé que des prédictions tirées de la recherche sur le changement linguistique - tel que la simplification d'un système casuel et des modifications dans l'ordre des mots - n'étaient pas soutenues par les données d'attrition (p. ex. Schmitt 2004 ; Schmid 2002). De telles réductions ne sont observables que dans les situations d'acquisition où l'input est réduit à certains contextes et/ou n'est pas entièrement conforme aux

\footnotetext{
${ }^{2}$ Sauf indication contraire, le présent article et les autres contributions dans ce numéro assument que les termes "attrition" ou "attrité" réfèrent à des bilingues tardifs, c'est-à-dire à des individus qui ont grandi de façon principalement monolingue (à l'exception d'une éventuelle expérience de l'enseignement d'une langue étrangère comme c'est aujourd'hui la règle dans la plupart des pays), et chez qui le début du bilinguisme et de l'attrition sont à situer au moment d'une émigration ayant eu lieu après la puberté.
} 
standards natifs, comme cela peut être le cas dans le changement intergénérationnel dans une situation de contact des langues.

De plus, les résultats cumulés d'un large éventail d'études de l'attrition ( $c f$. Köpke \& Schmid, ce volume) montrent clairement que les différences entre monolingues et attrités - dont la nature reste pourtant tout à fait intéressante - ne concernent qu'une petite proportion des contextes. Schmid (sous presse) propose que même pour les structures pour lesquelles l'attrition est avérée, celle-ci affecte généralement moins de $5 \%$ du total des occurrences. Cela suggère que l'attrition est le résultat de l'interaction (du moins partielle) de deux systèmes linguistiques durant le traitement du langage en production et en compréhension et n’affecte pas les connaissances linguistiques sous-jacentes. Ainsi, l'attrition apparaît comme phénomène psycholinguistique qui affecte le comportement langagier individuel, phénomène bien distinct du changement progressif ou de la détérioration qui surviennent notamment au niveau de la transmission d'une langue d'une génération à la suivante dans des groupes linguistiques, et qui peuvent affecter tout un système grammatical et même entraîner la disparition d'une langue.

Peu à peu il a donc été reconnu que l'acquisition d'une langue seconde est un corrélat plus pertinent pour l'attrition et que la recherche sur l'attrition peut non seulement bénéficier des fondements et des notions théoriques du développement bilingue mais également, dans une certaine mesure, contribuer à leur validation (cf. Köpke \& Schmid, ce volume). Cette contribution porte tout particulièrement sur la question de savoir si des différences entre données bilingues et monolingues (tant au niveau de la production que de la compréhension) se situent au niveau de la représentation des connaissances ou au niveau de l'accès à ces connaissances, une question qui est étroitement liée au débat sur la compétence et la performance. La définition de notions comme les connaissances linguistiques sous-jacentes et leur mise en œuvre avec les contraintes temporelles de la production et de la compréhension du langage en temps réel varient largement selon la conception théorique sur laquelle on s’appuie. La question de la « profondeur » à laquelle l'attrition peut affecter ces connaissances a ainsi des implications différentes selon le cadre théorique : ainsi un contexte théorique basé sur la présupposition d'une faculté innée d'acquisition d'une grammaire donne lieu à des prédictions différentes pour la perte d'une langue (basées sur l'idée que cette perte sera en accord avec des principes universaux sous-jacents) qu'un cadre théorique qui conçoit l'acquisition (et donc l'attrition) comme principalement dépendante de l’usage qui est fait de la langue. Dans les premières approches, il fait maintenant consensus de dire que l'attrition tardive — c'est-à-dire chez des locuteurs pour qui l'immersion dans un contexte où la L2 est parlée se situe après la puberté — se limite aux phénomènes d'interface et n'affecte pas la syntaxe étroite (p.ex. Sorace 2005). Dans le cadre d'orientations plus psycholinguistiques, on s'interroge moins sur la restructuration de la compétence sous-jacente et davantage sur le degré des difficultés d'accès à cette compétence. Ainsi on s’intéressera dans ce contexte à la question de savoir si le locuteur peut toujours accéder certaines structures ou items lexicaux pour la production verbale et si ce n'est pas le cas, si la structure ou l'item est au moins 
disponible pour la reconnaissance en compréhension (par exemple Hansen 2011; Paradis 2007). Dans ce contexte, la prise en compte de différents types de mémoire (procédurale/déclarative), de la fréquence distributionnelle et de l'exposition à la L1que le locuteur garde sont pertinentes. Toutefois, indépendamment de ces distinctions disciplinaires par rapport à la vision de la faculté de langage et de son utilisation, des données convergentes des études sur l'attrition suggèrent qu'une fois que le système de la L1 est acquis et stabilisé - un processus qui est généralement considéré comme accompli autour de l'âge de 12 ans (cf. Köpke \& Schmid, ce volume) - ce système, en lui-même, n’est plus vulnérable à l'attrition. C'est sans doute cette stabilité qui permet aux études de l'attrition d'approfondir le débat sur les « déficits représentationnels » dans la recherche sur l'acquisition des langues secondes (p. ex. Hawkins \& Hattori 2006 ; Snape, Leung \& Sharwood Smith 2009) : en effet, l'étude de l'attrition peut fournir des données comparatives caractérisées par le transfert translinguistique à l'exclusion de tout déficit représentationnel. Pour cette raison, il est essentiel que la recherche sur le bilinguisme se rende à l'évidence que l'affirmation de Grosjean ne signifie pas que le bilingue serait un monolingue et demi, mais plutôt qu'elle ou lui est différent d'un monolingue au niveau des deux systèmes linguistiques et que la comparaison entre systèmes linguistiques acquis à partir de la naissance et systèmes acquis plus tard dans la vie peut apporter des réponses valides à ce type de questionnements.

Les études rassemblées ici traitent de quelques-unes des questions que nous avons rapidement évoquées. Le premier article, de Köpke et Schmid, fournit une vue d'ensemble de l'état actuel de la recherche sur l'attrition, en développant notamment la délimitation du champ de l'attrition par rapport à d'autres champs de recherche comme le glissement linguistique à travers plusieurs générations et dans les situations de contact de langues, ainsi que par rapport à l'acquisition incomplète et l'effet du bilinguisme tout court. L’article présente ensuite une variété de facteurs neuropsycholinguistiques impliqués dans l'attrition et décrit quelques manifestations psycholinguistiques du processus d'attrition. Le point de vue défendu est celui de l'attrition de la L1 chez les bilingues tardifs en tant que phénomène avant tout psycholinguistique, témoignant de la compétition entre deux langues avec des états d'activation très différents.

Cette introduction est suivie de deux articles portant sur l'attrition du turc en tant que L1 et de deux articles où la L1 est l'espagnol. Gürel et Yilmaz étudient les propriétés du liage de pronoms sujets pleins et nuls chez des immigrés de langue maternelle turque. La recherche décrite ici réplique deux études plus anciennes de ces phénomènes dans une situation de contact avec l'anglais au Canada (Gürel 2002, 2004). La présente étude porte sur des immigrés turcs de la première et de la seconde génération aux Pays-Bas, ce qui implique des différences importantes entre les situations de migration étudiées : les Turcs aux Pays-Bas représentent un groupe de migrants beaucoup plus important qui a tendance à vivre dans des communautés plutôt unies. Cela a pour conséquence des taux de transmission de la L1 à la génération suivante plus élevés que dans le contexte nord-américain. Pourtant, les données des deux populations aux Pays-Bas (attrités et immigrés de la seconde 
génération) sont très similaires de celles observées par Gürel (2002, 2004) : tous les groupes bilingues montrent une certaine restructuration au niveau de l'interprétation des pronoms sujets pleins et nuls en comparaison avec le groupe contrôle monolingue. Ce qui est particulièrement intéressant, c’est que dans les cas où la L2 ne possède pas de forme grammaticale susceptible d'entrer en compétition avec la L1 (p.ex. le pronom plein kendisi et le pronom nul, pour lesquels les sujets du groupe de contrôle hésitent entre la référence liée et la référence disjointe), les bilingues semblent avoir tendance à recourir à l'interprétation canonique. Ce résultat est interprété comme un pattern de préférence grammaticale émergeant parmi les bilingues qui est compatible avec la GU (grammaire universelle). On trouve ces patterns dans des proportions similaires dans les réponses des immigrés de la première et de la seconde génération et autant chez les locuteurs de l’anglais que du néerlandais. Gürel et Yilmaz concluent que l'âge au moment de la migration ne semble pas avoir joué un rôle prédominant dans leurs données, ce qui est peut-être dans une certaine mesure lié à la grande vitalité dont bénéficie la langue turque aux Pays-Bas.

L'étude de Yilmaz porte sur une population d'attrités similaire : les bilingues tardifs turcs aux PaysBas. Elle examine dans quelle mesure des structures phrastiques syntaxiquement complexes, notamment les différents types d'enchâssement du turc, survivent à une longue période de résidence dans un contexte où la L2 est prédominante. En s’appuyant sur des recherches antérieures, elle classe les différentes formes d'enchâssement en fonction de leur complexité morphologique, c'est-à-dire selon le nombre de processus flexionnels requis. Elle constate d'après la comparaison à un groupe de locuteurs natifs quasi-monolingues en Turquie que les attrités ne diffèrent des sujets du groupe de contrôle que dans l'utilisation d'un seul type d'enchâssement, mais il s'agit de celui qui est morphologiquement le plus complexe. Des facteurs extralinguistiques comme la durée d'immigration, la fréquence d'utilisation de la L1, les orientations culturelles et le niveau de compétence en L2 ne permettent pas de prédire les variations individuelles, seul le niveau d'études joue un certain rôle. L’article de Montrul aborde une thématique qui a gagnée en importance dans la recherche sur l'attrition depuis l'étude-phare de Pallier et al. (2003), à savoir la question de ce qui est perdu et de ce qui est retenu d'une L1 chez des personnes adoptées dans un contexte international (cf. aussi Schmid, à paraître). Montrul présente l'étude du cas d'une femme adoptée au Guatemala par des parents américains qui ont fortement découragé toute utilisation de l'espagnol. A l'école secondaire et plus tard en tant que jeune adulte, elle a tenté de réapprendre sa langue de naissance qu'elle utilise maintenant fréquemment. Lors de l'étude de son comportement verbal au niveau d'une variété de variables morpho-syntaxiques, Montrul pose la question fascinante de savoir si son expérience interrompue avec cette langue a permis de lui fournir un avantage substantiel par rapport aux apprenants qui n’ont pas bénéficié d'une telle exposition précoce. Elle conclut que l’interruption de l'input dans la langue de naissance chez des adoptées en contexte international entraîne des conséquences radicales pour leur potentiel à développer entièrement cette langue, mais que l'âge est un facteur très important à ce niveau. La rétention et la facilité de réapprentissage sont fortement 
affectées par les attitudes et la motivation - ce qui est évidemment un sujet complexe chez les adoptés en général et de surcroît chez ceux qui ont été adoptés dans un contexte international.

Finalement, l'article de Perpiñán propose une contribution pour un débat qui a déjà entraîné des controverses dans la recherche sur l'attrition, à savoir la question de savoir si l'attrition est restreinte à des phénomènes d'interface où si elle peut aussi affecter la syntaxe étroite. Dans son étude d'un groupe de locuteurs hispanophones aux Etats-Unis qui utilisent l'anglais sur une base quotidienne, elle trouve des indices pour une optionalité émergente dans un type de construction avec inversion sujetverbe motivée par des contraintes pragmatiques, mais pas dans les contextes ou l'inversion est motivée par des contraintes purement syntaxiques. Ces résultats soutiennent l'hypothèse de l' Optionality at the Interfaces Hypothesis proposée par Sorace (2005). Par ailleurs, la compréhension de ces structures et les temps de réponse ne sont pas affectés dans ces données.

Dans l'ensemble, ces contributions montrent que la recherche sur l'attrition peut contribuer à éclairer quelques-unes des questions principales soulevées par la recherche sur l'acquisition d'une langue seconde, comme par exemple le débat autour de l’hypothèse de la période critique, la question du déficit représentationnel, l'hypothèse de l'interface, etc. De plus, plusieurs des contributions montrent que la dimension psycholinguistique de l'attrition ne se limite pas à des difficultés dans l'accès au lexique, mais peut également affecter des traitements morpho-syntaxiques dans certains domaines spécifiques. 


\section{REFERENCES}

Boyd, S. 1986. Using the present to predict the future in language contact: the case of immigrant minority languages in Sweden. In B. Weltens, K. de Bot \& T. van Els (Eds.), Language attrition in progress, 99-115. Dordrecht: Foris.

Dressler, W.U. 1991. The sociolinguistic and patholinguistic attrition of Breton phonology, morphology, and morphonology. In H.W. Seliger and R.M. Vago (Eds.), First language attrition, 99-112. Cambridge: Cambridge University Press.

Flege, J. E. (1987). The production of 'new' and 'similar' phones in a foreign language: evidence for the effect of equivalence classification. Journal of Phonetics $n^{\circ} 15,47-65$.

Grosjean, F. (1989). Neurolinguists, beware! The bilingual is not two monolinguals in one person. Brain and Language $\mathrm{n}^{\circ}$ 36(1), 3-15.

Gürel, A. (2002). Linguistic characteristics of second language acquisition and first language attrition: overt versus null pronouns. Unpublished Doctoral Dissertation, McGill University, Montreal, Canada.

Gürel, A. (2004). Attrition in L1 competence: the case of Turkish. In M.S. Schmid, B. Köpke, M. Keijzer \& L. Weilemar (Eds.), First language attrition: interdisciplinary perspectives on methodological issues, 225-242. Amsterdam: John Benjamins.

Hansen, L. (2011). The acquisition, attrition, and relearning of mission vocabulary. In M. Schmid \& W. Lowie (Eds.), Modeling Bilingualism: From structure to chaos, 115-134. Amsterdam: John Benjamins.

Hawkins, R. \& Hattori, H. (2006). Interpretation of English multiple wh-questions by Japanese speakers: A missing uninterpretable feature account. Second Language Research $\mathrm{n}^{\circ} 22,269-301$.

Huffines, M.L. (1991). Pennsylvania German: convergence and change as strategies of discourse. In H.W. Seliger \& R.M. Vago (Eds.), First language attrition, 125-138. New York, NY: Cambridge University Press.

Pallier, C., Dehaene, S., Poline, J.-B., LeBihan, D., Argenti, A.-M., Dupoux, E. \& Mehler, J. (2003). Brain imaging of language plasticity in adopted adults: Can a second language replace the first ? Cerebral Cortex n 13(2), 155-161.

Paradis, M. (2007). L1 attrition features predicted by a neurolinguistic theory of bilingualism. In B. Köpke, M.S. Schmid, M. Keijzer \& S. Dostert (Eds.), Language Attrition: theoretical perspectives, 121-133. Amsterdam: John Benjamins.

Schmid, M.S. (2002). First language attrition, use and maintenance: the case of German Jews in anglophone countries. Amsterdam: John Benjamins.

Schmid, M.S. forthcoming. Language attrition. Linguistic Approaches to Bilingualism.

Schmitt, E. (2004). No more reductions! To the problem of evaluation of language attrition data. In M. S. Schmid, B. Köpke, M. Keijzer \& L. Weilemar (Eds.), First language attrition: interdisciplinary perspectives on methodological issues, 299-316. Amsterdam: John Benjamins. 
Seliger, H.W. \& Vago, R.M. (Eds.). 1991. First language attrition. Cambridge: Cambridge University Press.

Snape, N., Leung, Y.I. \& Sharwood Smith, M. (Eds.) (2009). Representational deficits in SLA: studies in honour of Roger Hawkins. Amsterdam: John Benjamins.

Sorace, A. (2005). Selective optionality in language development. In L.E.A. Cornips \& K.P. Corrigan (Eds.), Syntax and variation: reconciling the biological and the social, 55-80. Amsterdam: John Benjamins.

Weltens, B., de Bot, K. \& van Els, T. (Eds.). 1986. Language attrition in progress. Dordrecht: Foris. 